\title{
RANKS OF JACOBIANS IN TOWERS OF FUNCTION FIELDS
}

\author{
Douglas Ulmer and Yuri G. Zarhin
}

\section{Introduction}

Let $k$ be a field of characteristic zero and $k(t)$ the rational function field over $k$. Several authors have considered the behavior of ranks of elliptic curves in towers of function fields such as $k\left(t^{1 / n}\right)$. See for example [Shi86], [Ulm02], [Sil04], [Ell06], [Ulm07a], [Ulm07b], [Ber08], and [UlmDPCT]. In particular, it is known that there are elliptic curves $E$ over $k(t)$ such that the rank of $E(K)$ stays bounded as $K$ varies through the tower of function fields $K=k\left(t^{1 / p^{r}}\right)$ with $r \geq 1$ or the tower $K=k\left(t^{1 / d}\right)$ with $d \geq 1$.

In this paper we combine the rank formula of [UlmDPCT] with strong upper bounds on endomorphisms of Jacobians due to the senior author (e.g., [Zar05]) to give many examples of higher dimensional, absolutely simple Jacobians over $k(t)$ with bounded rank in towers $k\left(t^{1 / p^{r}}\right)$. In many cases we are able to compute the rank at every layer of the tower.

Our methods give rise to many examples, but to fix ideas we state the following typical result.

1.1. Theorem. Let $g_{X}$ be an integer $\geq 2$ and let $X$ be the smooth, proper curve of genus $g_{X}$ over $\mathbb{Q}(t)$ with affine plane model

$$
t y^{2}=x^{2 g_{X}+1}-x+t-1 .
$$

Let $J$ be the Jacobian of $X$. Then $J$ is absolutely simple and the $\overline{\mathbb{Q}(t)} / \overline{\mathbb{Q}}$-trace of $J$ is zero. Moreover, for every prime number $p$ and every integer $r \geq 0$, we have

$$
\operatorname{Rank} J\left(\overline{\mathbb{Q}}\left(t^{1 / p^{r}}\right)\right)=2 g_{X} .
$$

\section{Endomorphism algebras of superelliptic Jacobians}

2.1. Notation. In this section, we collect some results on the endomorphism algebras of certain superelliptic Jacobians. Throughout the paper, $\bar{k}$ will denote an algebraic closure of $k$.

If $X$ and $Y$ are abelian varieties over $k$, then we write $\operatorname{End}(X)$ and $\operatorname{Hom}(X, Y)$ for the corresponding ring and group of homomorphisms over $\bar{k}$.

Let $f$ be a non-constant polynomial with coefficients in $k$ and without multiple roots. We write $m$ for the degree of $f, \mathcal{R}_{f}$ for the set of roots of $f$ in $\bar{k}$, and $k\left(\mathcal{R}_{f}\right)$ for the splitting field of $f$. As usual

$$
\operatorname{Gal}(f)=\operatorname{Aut}\left(k\left(\mathcal{R}_{f}\right) / k\right) .
$$

Received by the editors February 17, 2010. 
For example, consider $f_{m}=x^{m}-x-1$. It is known [Ser08, p. 42] that if $k=\mathbb{Q}$ then $\operatorname{Gal}\left(f_{m}\right)$ is the symmetric group $S_{m}$.

2.2. Superelliptic Jacobians. Given a polynomial $f$ of degree $m$ with distinct roots and a positive integer $d$, let $C_{f, d}$ be the smooth projective curve over $k$ with affine model

$$
z^{d}=f(x) .
$$

Let $J_{f, d}$ be the Jacobian of $C_{f, d}$. In this paper we will only consider the case where $d=q=p^{r}$ with $p$ a prime number. To avoid trivialities, we always assume that $m>1$ and $q>1$.

We have an obvious projection $C_{f, q} \rightarrow C_{f, q / p}$ and an induced homomorphism of Jacobians $J_{f, q} \rightarrow J_{f, q / p}$. Since $C_{f, q} \rightarrow C_{f, q / p}$ is totally ramified over the zeroes of $f$, the homomorphism $J_{f, q} \rightarrow J_{f, q / p}$ has connected kernel, which we call the new part of $J_{f, q}$ and which we denote $J^{(f, q)}$. It is easy to see that $J_{f, q}$ is isogenous to $\prod_{i=1}^{r} J^{\left(f, p^{i}\right)}$.

A simple application of the Riemann-Hurwitz formula shows that

$$
\operatorname{dim}\left(J_{f, q}\right)=(m-1)(q-1) / 2-(\operatorname{gcd}(q, m)-1) / 2
$$

and

$$
\operatorname{dim}\left(J^{(f, q)}\right)= \begin{cases}(m-1) \phi(q) / 2 & \text { if } q \nmid m \\ (m-2) \phi(q) / 2 & \text { if } q \mid m .\end{cases}
$$

Note in particular that $J^{(f, q)}$ is non-zero except when $m \leq 2$ and $q \leq 2$.

We write $\zeta_{q}$ for a primitive $q$-th root in $\bar{k}$. Over $k\left(\zeta_{q}\right)$, we have a natural action of the $q$-th roots of unity on $C_{f, q}$, namely $(x, z) \mapsto\left(x, \zeta_{q} z\right)$. The induced action on Jacobians gives rise to a homomorphism $\mathbb{Z}\left[\zeta_{q}\right] \rightarrow \operatorname{End}\left(J^{(f, q)}\right)$ which is known to be injective whenever $J^{(f, q)}$ is non-zero. (See [Zar05, 4.8], [Zar07, 5.8], and [Xue, 2.6]; see also [UlmDPCT, 7.8.1] for this result in a slightly more general context.) We will be interested in various conditions which guarantee that this homomorphism is an isomorphism.

2.3. The case $m=2$. Suppose that $m=2$, i.e., that $f$ is quadratic. By a linear change of variable we may take $f$ to have the form $f(x)=x^{2}-a$ where $a \in k$ is nonzero. If $q>2$ (so that $J^{(f, q)}$ is non-zero), we have $\operatorname{dim}\left(J^{(f, q)}\right)=\phi(q) / 2$. The inclusion $\mathbb{Z}\left[\zeta_{q}\right] \hookrightarrow \operatorname{End}\left(J^{(f, q)}\right)$ exhibits a commutative integral domain of $\operatorname{rank} 2 \operatorname{dim}\left(J^{(f, q)}\right)$ as a subalgebra of $\operatorname{End}\left(J^{(f, q)}\right)$ and so over $\bar{k}, J^{(f, q)}$ is an abelian variety of CM type. By [Shi98, Prop. 3, p. 36], $J^{(f, q)}$ is isogenous to a power $A^{e}$ of a simple abelian variety $A$ of CM type. It may happen (for example if $q$ is even) that $e>1$ in which case $\operatorname{End}\left(J^{(f, q)}\right)$ is strictly bigger than $\mathbb{Z}\left[\zeta_{q}\right]$. In any case, it follows that $J_{f, q}$ is of CM type over $\bar{k}$.

2.4. An alternative approach to the case $f(x)=x^{2}-a$ that works more generally for $f(x)=x^{m}-a$ is to note that the curve $z^{d}=x^{m}-a$ is obviously covered (over $\bar{k}$ ) by the Fermat curve of degree $\operatorname{lcm}(m, d)$. Since the Jacobian of the Fermat curve is an abelian variety of CM type [Sch84, VI, 1.2 and 1.5], the same is true of $J_{f, d}$ for all $d$. 
2.5. The case $m \geq 5$. For $m>2$ it is again not true in general that $\mathbb{Z}\left[\zeta_{q}\right] \cong$ $\operatorname{End}\left(J^{(f, q)}\right)$. However, one expects that for "sufficiently general" $f, J^{(f, q)}$ should have no endomorphisms beyond the obvious ones. We make this precise in several cases, starting with $m \geq 5$.

2.5.1. Theorem. Suppose that $m \geq 5$ and $\operatorname{Gal}(f)=S_{m}$ or $A_{m}$. Then

$$
\operatorname{End}\left(J^{(f, q)}\right)=\mathbb{Z}\left[\zeta_{q}\right]
$$

In particular, $J^{(f, q)}$ is an absolutely simple abelian variety that is not of CM type over $\bar{k}$.

Proof. The endomorphism ring was calculated in earlier works of Zarhin and Xue: The case when either $p$ does not divide $m$ or $q$ divides $m$ is proven in [Zar05, Th. 4.17 on p. 359]. The remaining case when $p$ divides $m$ but $q$ does not divide $m$ is proven in [Xue]. Since the endomorphism ring is a domain, $J^{(f, q)}$ is absolutely simple. Since $m \geq 5$, the dimension of $J^{(f, q)}$ is strictly greater than $\phi(q) / 2$ and so $J^{(f, q)}$ is not of CM type.

2.6. The cases $m=4$ and $m=3$. These cases (which are not needed for the basic Theorem 1.1) are more complicated and require additional hypotheses.

2.6.1. Theorem. Suppose that $p$ is odd, $m=4$, and $\operatorname{Gal}(f)=S_{4}$. Let $k^{\prime}$ be the unique subextension of $k\left(\mathcal{R}_{f}\right)$ which is quadratic over $k$. Assume that $k^{\prime}$ does not lie in $k\left(\zeta_{p}\right)$. Then the Galois group of $f(x)$ over $k\left(\zeta_{q}\right)$ is still $S_{4}$ and

$$
\operatorname{End}\left(J^{(f, q)}\right)=\mathbb{Z}\left[\zeta_{q}\right]
$$

In particular, $J^{(f, q)}$ is an absolutely simple abelian variety that is not of CM type over $\bar{k}$.

Proof. In light of [Zar09, Cor. 1.5 on p. 693 and Cor. 5.3 on p. 705], it suffices to check that the Galois group of $f(x)$ over $k\left(\zeta_{q}\right)$ remains $S_{4}$, i.e., that $k\left(\mathcal{R}_{f}\right)$ and $k\left(\zeta_{q}\right)$ are linearly disjoint over $k$. Since $p$ is odd, $\operatorname{Gal}\left(k\left(\zeta_{q}\right) / k\right) \cong G_{2} \times G_{\text {odd }}$ where $G_{2}$ is a cyclic group of 2-power order and $G_{\text {odd }}$ is a cyclic group of odd order. Moreover $k\left(\zeta_{q}\right)^{G_{\text {odd }}} \subset k\left(\zeta_{p}\right)$. Since $S_{4}$ is generated by transpositions, it has no non-trivial quotients of odd order. Thus $k\left(\mathcal{R}_{f}\right)$ and $k\left(\zeta_{q}\right)$ are linearly disjoint if and only if $k^{\prime}$ and $k\left(\zeta_{p}\right)$ are linearly disjoint (which in turn occurs if and only if $k^{\prime}$ and $k\left(\zeta_{q}\right)^{G_{\text {odd }}}$ are linearly disjoint).

Ramification conditions give a convenient criterion for linear disjointness:

2.6.2. Lemma. Let $L / \mathbb{Q}$ be a finite extension. Suppose that a prime $p$ is unramified in $L$. Then $L$ and $\mathbb{Q}\left(\zeta_{q}\right)$ are linearly disjoint over $\mathbb{Q}$.

Proof. This follows immediately from the fact that $\mathbb{Q}\left(\zeta_{q}\right) / \mathbb{Q}$ is totally ramified over $p$.

2.6.3. Theorem. Suppose that $k=\mathbb{Q}, m=4$ and $\operatorname{Gal}(f)=S_{4}$. If $p$ is an odd prime that is unramified in $\mathbb{Q}\left(\mathcal{R}_{f}\right)$ then

$$
\operatorname{End}\left(J^{(f, q)}\right)=\mathbb{Z}\left[\zeta_{q}\right]
$$

In particular, $J^{(f, q)}$ is an absolutely simple abelian variety that is not of CM type over $\bar{k}$. 
Proof. It follows from Lemma 2.6.2 that $\mathbb{Q}\left(\mathcal{R}_{f}\right)$ and $\mathbb{Q}\left(\zeta_{p}\right)$ are linearly disjoint over $\mathbb{Q}$. Theorem 2.6.1 then implies that the endomorphism ring is $\mathbb{Z}\left[\zeta_{q}\right]$. Since this is a domain, $J^{(f, q)}$ is absolutely simple and since $\operatorname{dim} J^{(f, q)}>\phi(q) / 2$, it is not of CM type.

2.6.4. Examples. Let $k=\mathbb{Q}$ and $f(x)=f_{4}(x)=x^{4}-x-1$. Then the discriminant of $f_{4}(x)$ is -283 (see [Zar09, p. 693]). Since 283 is a prime, it follows that $\mathbb{Q}\left(\mathcal{R}_{f_{4}}\right) / \mathbb{Q}$ is unramified outside 283. So, if $p$ is odd and $\neq 283$ then for all $q=p^{r}$

$$
\operatorname{End}\left(J^{\left(f_{4}, q\right)}\right)=\mathbb{Z}\left[\zeta_{q}\right]
$$

(The case of $q=p=3$ is Example 1.6 of [Zar09].)

For another example, again take $k=\mathbb{Q}$ and let $f=x^{4}-x+2$. The discriminant of $f$ is $-27 \cdot(-1)^{4}+256 \cdot 2^{3}=2021=43 \cdot 47$. Reducing mod 3 , one checks that $f$ has no roots in $\mathbb{F}_{3}$ nor in $\mathbb{F}_{9}$ and so is irreducible over $\mathbb{F}_{3}$ and, a fortiori, irreducible over $\mathbb{Q}$. Moreover, the Galois group $\operatorname{Gal}(f)$ is isomorphic to $S_{4}$. (Indeed, looking at the Frobenius at $p$ for $p=2,3,5$ shows that $\operatorname{Gal}(f)$ contains a transposition, a 4 -cycle, and a 3 -cycle. Since the only subgroups of $S_{4}$ generated by a 4-cycle and a transposition are all of $S_{4}$ or a group of order 8 , we see that $\operatorname{Gal}(f)$ must be $S_{4}$.) The argument of the first example applies when $p$ is odd and $\neq 43,47$. But since $p=43$ and $p=47$ are congruent to $3 \bmod 4$, the quadratic subfield of $\mathbb{Q}\left(\zeta_{p}\right)$ is imaginary for these $p$. On the other hand, since $2021>0$, the unique quadratic subfield of $\mathbb{Q}\left(\mathcal{R}_{f}\right)$ is real. Thus we have linear disjointness for all $p$. It follows that

$$
\operatorname{End}\left(J^{\left(f_{4}, q\right)}\right)=\mathbb{Z}\left[\zeta_{q}\right]
$$

for all odd $p$.

2.6.5. Theorem (Theorem 5.17 of [Zar07]). Suppose that $m=3, p=2$, and $q=4$. Then $\operatorname{End}\left(J^{(f, q)}\right)$ is an order in the matrix algebra of size two over the imaginary quadratic field $\mathbb{Q}\left(\zeta_{4}\right)=\mathbb{Q}(\sqrt{-1})$.

2.6.6. Theorem (Theorem 5.18 of [Zar07]). Suppose that $k_{0}$ is an algebraically closed field of characteristic zero and $k=k_{0}(z)$ is the field of rational functions in the variable $z$. Suppose that $m=3$ or 4 and $\operatorname{Gal}(f)=S_{m}$. If $(m, q) \neq(3,4)$ then

$$
\operatorname{End}\left(J^{(f, q)}\right)=\mathbb{Z}\left[\zeta_{q}\right]
$$

2.6.7. Example. Let $k_{0}=\overline{\mathbb{Q}} \subset \mathbb{C}$ and $f(x)=x^{3}-x-z$. One may view $\overline{\mathbb{Q}}(z)$ as a subfield of $\mathbb{C}$, sending $z$ to any transcendental complex number $\beta$. Then we get the complex polynomial $f_{3, \beta}(x)=x^{3}-x-\beta \in \mathbb{C}[x]$ and conclude that for each $q \neq 4$ the complex abelian variety $J^{\left(f_{3, \beta}, q\right)}$ satisfies

$$
\operatorname{End}\left(J^{\left(f_{3, \beta}, q\right)}\right)=\mathbb{Z}\left[\zeta_{q}\right]
$$

\section{Homomorphisms between superelliptic Jacobians}

We can apply the results of the previous section to obtain pairs of superelliptic Jacobians with no homomorphisms between them. 
3.1. The case $g=y^{n}-a$. In this section, we assume that $n \geq 2$ and $g(y)=y^{n}-a$ for some non-zero $a$ in $k$. By Section 2.4, for all powers $q$ of a prime $p$ with $q>2$, $J_{g, q}$ is an abelian variety of CM type.

3.1.1. Theorem. Let $q=p^{r}$ for a prime number $p$. Suppose that $m \geq 4, f$ is an irreducible polynomial over $k$ of degree $m$, and the Galois group $\operatorname{Gal}(f)=S_{m}$ or $A_{m}$. If $m=4$ then we also assume that $p$ is odd, $\operatorname{Gal}(f)=S_{4}$, and at least one of the following two conditions holds:

(i) the unique quadratic subextension of $k\left(\mathcal{R}_{f}\right)$ does not lie in $k\left(\zeta_{p}\right)$.

(ii) $k=\mathbb{Q}$ and $p$ is unramified in $\mathbb{Q}\left(\mathcal{R}_{f}\right)$.

Then $\operatorname{Hom}\left(J\left(C_{f, q}\right), J\left(C_{g, q}\right)\right)=\{0\}$.

Proof. It suffices to check that $\operatorname{Hom}\left(J^{\left(f, p^{i}\right)}, J\left(C_{g, q}\right)\right)=\{0\}$ for all positive $i \leq r$. By Theorems 2.5.1, 2.6.1 and 2.6.3, $J^{\left(f, p^{i}\right)}$ is absolutely simple and not of CM type over $\bar{k}$. But $J\left(C_{g, q}\right)$ is of CM type over $\bar{k}$.

3.2. The case $m>n \geq 4$.

3.2.1. Theorem. Suppose that $m>n \geq 4$, the Galois groups $\operatorname{Gal}(f)=S_{m}$ or $A_{m}$ and $\operatorname{Gal}(g)=S_{n}$ or $A_{n}$. If $n=4$ then we also assume that $p$ is odd, $\operatorname{Gal}(g)=S_{4}$, and at least one of the following two conditions holds:

(i) the unique quadratic subextension of $k\left(\mathcal{R}_{g}\right)$ does not lie in $k\left(\zeta_{p}\right)$.

(ii) $k=\mathbb{Q}$ and $p$ is unramified in $\mathbb{Q}\left(\mathcal{R}_{g}\right)$.

Then $\operatorname{Rank} \operatorname{Hom}\left(J_{f, q}, J_{g, q}\right)$ is bounded by a constant independent of $p$ and $q$.

Proof. It will suffice to show that

$$
\operatorname{Hom}\left(J^{\left(f, q_{1}\right)}, J^{\left(g, q_{2}\right)}\right)=\{0\}
$$

for all divisors $q_{1}$ and $q_{2}$ of $q$ except possibly when $q_{1}=q_{2}$ and $q_{1} \mid m$. Under the hypotheses of the theorem, Theorems 2.5.1, 2.6.1 and 2.6.3 imply that $J^{\left(f, q_{1}\right)}$ and $J^{\left(g, q_{2}\right)}$ are absolutely simple with endomorphism algebras $\mathbb{Q}\left(\zeta_{q_{1}}\right)$ and $\mathbb{Q}\left(\zeta_{q_{2}}\right)$ respectively. Thus either

$$
\operatorname{Hom}\left(J^{\left(f, q_{1}\right)}, J^{\left(g, q_{2}\right)}\right)=\{0\}
$$

or $J^{\left(f, q_{1}\right)}$ and $J^{\left(g, q_{2}\right)}$ are isogenous. But if they were isogenous, comparing endomorphism algebras we would have $\phi\left(q_{1}\right)=\phi\left(q_{2}\right)$ and therefore $q_{1}=q_{2}$. Comparing dimensions (cf. Section 2.2), we would also have $n=m-1$ and $q_{1} \mid m$. Thus

$$
\operatorname{Hom}\left(J^{\left(f, q_{1}\right)}, J^{\left(g, q_{2}\right)}\right)=\{0\}
$$

unless $q_{1}=q_{2}$ and $q_{1} \mid m$.

\section{Berger curves}

4.1. Notation. Let $K=\bar{k}(t)$ be the rational function field over $\bar{k}$. Recall that given two rational functions $f$ and $g$ on $\mathbb{P}^{1}$ over $k$, under mild hypotheses Lisa Berger's construction [Ber08] gives a smooth proper curve $X_{f, g}$ over $K$ which is a model of the curve

$$
\{f-t g=0\} \subset \mathbb{P}_{K}^{1} \times \mathbb{P}_{K}^{1}
$$


Again under mild hypotheses, Berger computes the genus of $X_{f, g}$, shows that $X_{f, g}$ is absolutely irreducible, and that in a suitable sense it is associated to a tower of surfaces that are dominated by a product of curves.

More precisely, applying [Ber08, Thm. 3.1] we have:

4.2. Theorem. Let $k$ be a field of characteristic zero and let $K=\bar{k}(t)$. Let $f(x)$ and $g(y)$ be polynomials over $k$ of degrees $m$ and $n$ respectively with simple roots. Let $X_{f, g}$ be the smooth proper model of

$$
\{f-t g=0\} \subset \mathbb{P}_{K}^{1} \times \mathbb{P}_{K}^{1}
$$

over $K$. Then $X_{f, g}$ is absolutely irreducible and has genus

$$
g_{X}=\frac{(m-1)(n-1)-\operatorname{gcd}(m, n)+1}{2} .
$$

Let $J$ be the Jacobian of $X_{f, g}$, an abelian variety of dimension $g_{X}$ over $K$. For each positive integer $d$, let $K_{d}=\bar{k}\left(t^{1 / d}\right)$. Building on [Ber08], Ulmer gives a formula in [UlmDPCT] for the rank of the Mordell-Weil group $J\left(K_{d}\right)$ in terms of homomorphisms between $J_{f, d}$ and $J_{g, d}$.

More precisely, we have:

4.3. Theorem. With notation as above, for all $d$ the $K_{d} / \bar{k}$-trace of $J$ is zero. Moreover, we have

$$
\operatorname{Rank} J\left(K_{d}\right)=\operatorname{Rank} \operatorname{Hom}\left(J_{f, d}, J_{g, d}\right)^{\mu_{d}}-c_{1} d+c_{2}(d) .
$$

Here the exponent signifies those homomorphisms commuting with the natural action of $\mu_{d}$ on both factors, $c_{1}$ is a non-negative integer, and $c_{2}$ is a periodic function. We have

$$
c_{2}(d)=(m-1)(n-1)+\operatorname{gcd}(m, n, d)-1 .
$$

If for some $d$ strictly larger than $c_{2}(d)$ we have $\operatorname{Hom}\left(J_{f, d}, J_{g, d}\right)^{\mu_{d}}=0$, then $c_{1}=0$.

Proof. The vanishing of the $K_{d} / \bar{k}$ trace follows from [UlmDPCT, 5.6]. The formula for the rank is [UlmDPCT, 6.2] and the definition of $c_{2}$ in [UlmDPCT, 6.1.1] immediately gives the expression above. Since $c_{1}$ is non-negative and the right hand side of the rank formula is also non-negative, if the Hom group is zero for a large value of $d$, we must have $c_{1}=0$.

\section{Bounded ranks}

We now assemble the pieces to give several examples of Jacobians over $k(t)$ with bounded ranks in the tower $\bar{k}\left(t^{1 / p^{r}}\right)$. We treat only the most straightforward examples and the reader will have no trouble seeing that there are several other ways to apply the basic results of Sections 2 and 4 .

5.1. Proof of Theorem 1.1. For an integer $g_{X} \geq 2$, let $m=2 g_{X}+1, f(x)=$ $f_{m}(x)=x^{m}-x-1, n=2$, and $g(y)=y^{2}-1$. Then Berger's curve $X_{f, g}$ is a smooth, proper model of the hyperelliptic curve

$$
t y^{2}=x^{2 g_{X}+1}-x-1+t
$$

of genus $g_{X}$. Let $J$ be the Jacobian of $X_{f, g}$. Taking the ground field $k$ to be $\mathbb{Q}$, we know (see Section 2.1) that $f$ has Galois group $S_{m}$. Let $p$ be a prime number and $q$ a 
power of $p$. Applying Theorem 3.1.1, we have that $\operatorname{Hom}\left(J_{f, q}, J_{g, q}\right)=0$ and therefore by Theorem 4.3 , the $\overline{\mathbb{Q}}\left(t^{1 / q}\right) / \overline{\mathbb{Q}}$-trace of $J$ is zero and

$$
\operatorname{Rank} J\left(\overline{\mathbb{Q}}\left(t^{1 / q}\right)\right)=(m-1)(n-1)=2 g_{X} .
$$

Note that $X_{f, g}$ is a quadratic twist of the hyperelliptic curve

$$
X^{\prime}: \quad y^{2}=x^{2 g_{X}+1}-x-1+t
$$

and therefore $J$ is isomorphic over $\overline{\mathbb{Q}(t)}$ to the Jacobian $J^{\prime}$ of $X^{\prime}$; in particular, $\operatorname{End}\left(J^{\prime}\right)=\operatorname{End}(J)$. Clearly, the Galois group of $x^{2 g_{X}+1}-x-1+t$ over $\mathbb{Q}(t)$ coincides with the Galois group of $x^{2 g_{X}+1}-x-t$ over $\mathbb{Q}(t)$ and therefore equals $S_{m}$ (even over $\overline{\mathbb{Q}}(t))$ [Ser08, Thm. 4.4.1 on p. 39]. The case $q=2$ and $k=\mathbb{Q}(t)$ of Theorem 2.5.1 implies $\operatorname{End}\left(J^{\prime}\right)=\mathbb{Z}$ and therefore $\operatorname{End}(J)=\mathbb{Z}$. This shows in particular that $J$ is absolutely simple.

It follows from Theorem 5.18(i) on p. 384 of [Zar07] that in fact the $\overline{\mathbb{Q}(t)} / \overline{\mathbb{Q}}$-trace of $J$ is zero.

This completes the proof of Theorem 1.1.

5.2. Essentially identical arguments apply with $f(x)=x^{2 g_{X}+2}-x-1$ and $g=y^{2}-1$ and show that the Jacobian $J$ of the hyperelliptic curve

$$
t y^{2}=x^{2 g_{X}+2}-x-1+t
$$

is absolutely simple, has trivial $\overline{\mathbb{Q}}\left(t^{1 / q}\right) / \overline{\mathbb{Q}}$-trace, and has rank

$$
\operatorname{Rank} J\left(\overline{\mathbb{Q}}\left(t^{1 / q}\right)\right)=2 g_{X}+\operatorname{gcd}(q, 2)-1 .
$$

The vanishing of the $\overline{\mathbb{Q}(t)} / \overline{\mathbb{Q}}$-trace of $J$ follows from Theorem 1.2 of [Zar03] combined with Lemma 3.4 on p. 369 of [Zar07].

5.3. A superelliptic generalization. Consider the case $n \geq 2, g=y^{n}-1, m \geq 5$, $f=x^{m}-x-1$, and $k=\mathbb{Q}$. Berger's curve $X_{f, g}$ is the superelliptic curve

$$
t y^{n}=x^{m}-x-1+t
$$

of genus

$$
g_{X}=\frac{(m-1)(n-1)-\operatorname{gcd}(m, n)+1}{2} .
$$

Let $J$ be the Jacobian of $X_{f, g}$ and let $q$ be a power of a prime number $p$. Applying Theorem 4.3 and Theorem 3.1.1, we have that

$$
\operatorname{Rank} J\left(\overline{\mathbb{Q}}\left(t^{1 / q}\right)\right)=(m-1)(n-1)+\operatorname{gcd}(m, n, q)-1 .
$$

Note that this differs from $2 g_{X}$ by an amount bounded independently of $q$.

5.4. More examples. Now consider the case where $m$ and $n$ satisfy $m>n>4$ and $f, g$ and $p$ satisfy the hypotheses of Theorem 3.2.1. Berger's curve $X_{f, g}$ is as usual the smooth proper model of $f-t g=0$. It has genus

$$
g_{X}=\frac{(m-1)(n-1)-\operatorname{gcd}(m, n)+1}{2} .
$$

Let $J$ be the Jacobian of $X_{f, g}$. For every power $q$ of $p$, we have

$$
\operatorname{Rank} J\left(\bar{k}\left(t^{1 / q}\right)\right) \leq(m-1)(n-1)+\operatorname{gcd}(m, n, d)-1+\epsilon
$$

where $\epsilon$ is a constant which is independent of $p$ and $q$. 
5.5. Infinitely many non-isogenous examples over a Hilbertian field. Let us discuss a generalization of previous examples that is based on the notion of Morse polynomial [Ser08, p. 39]. (Notice that $x^{m}-x$ is a Morse polynomial and $f_{m}(x)=$ $\left(x^{m}-x\right)-1$.) Namely, let $h(x) \in k[x]$ be a Morse polynomial of degree $m \geq 5$. Then the Galois group of the polynomial of $h(x)-t$ over $k(t)$ is $S_{m}$ [Ser08, Thm. 4.4.1 on p. 39]. Let us consider the subset $A(h) \subset k$ consisting of all $c \in k$ such that the Galois group of $h(x)-c$ over $k$ is $S_{m}$. Assuming $c \in A(h)$, let $f(x)=h(x)-c$ and $g(y)=y^{2}-1$. The hyperelliptic curve

$$
X_{h-c, g}: \quad h(x)-c-t\left(y^{2}-1\right)=0
$$

which is a $k(\sqrt{t}) / k(t)$-twist of

$$
X_{c}^{\prime}: \quad y^{2}=h(x)-c+t=h(x)-(-t+c)
$$

has genus $\left[\frac{n-1}{2}\right]$. Clearly, the Galois group of $h(x)-(-t+c)$ over $k(t)$ is also $S_{m}$; in particular, by Theorem 2.5.1, the Jacobian $J_{c}$ of $X_{h-c, g}$ has zero $\bar{k}(t) / \bar{k}$-trace and $\operatorname{End}(J)=\mathbb{Z}$. Arguing as in the proof of Theorem 1.1, we have that the rank of $J_{c}\left(\bar{k}\left(t^{1 / p^{r}}\right)\right)$ is bounded independently of $p$ and $r$. Let $J_{c}^{\prime}$ be the Jacobian of $X_{c}^{\prime}$ and note that $J_{c}^{\prime}$ and $J_{c}$ become isomorphic over $k\left(t^{1 / 2}\right)$.

Now suppose that $d$ is a different element of $A(h)$; assume in addition that the splitting field $k_{c}$ of $h(x)-c$ and the splitting field $k_{d}$ of $h(x)-d$ are linearly disjoint over $k$. Let us consider the Jacobians $J_{c}^{\prime}$ and $J_{d}^{\prime}$ as abelian varieties over the complete discrete valuation field $k((t))$. Clearly, they both have good reduction at $t=0$; their reductions are the Jacobians of $y^{2}=h(x)-c$ and $y^{2}=h(x)-d$ respectively. In addition, the field of definition of points of order 2 on $J_{c}^{\prime}$ (resp. on $J_{d}^{\prime}$ ) coincides with $k_{c}((t))$ (resp. with $k_{d}((t))$; in particular, those splitting fields are linearly disjoint over $k((t))$. Now it follows from Theorem 1.2 of [Zar03] that $J_{c}^{\prime}$ and $J_{d}^{\prime}$ are not isogenous over $\overline{k((t))}$ ! Therefore $J_{c}$ and $J_{d}$ are not isogenous over $\overline{k(t)}$.

Notice that if $k$ is Hilbertian (e.g., a number field) then Hilbert's irreducibility theorem guarantees the existence of plenty of such $c$ and $d$.

5.6. One more elliptic example. As in Example 2.6.7, let $k=\mathbb{C}$ and let $\beta$ be a transcendental complex number. If we put $g(x)=f(x)=x^{3}-x-\beta$ then Berger's construction gives us an an elliptic curve $X_{f, g}:\left(x^{3}-x-\beta\right)-t\left(y^{3}-y-\beta\right)$ over the function field $\mathbb{C}(t)$ that appears (in slightly disguised form) in [UlmDPCT, Sect. 9]. Applying Theorem 2.6.6 and the rank formula in [UlmDPCT, Section 9.3] we have that the rank of $X_{f, g}\left(\mathbb{C}\left(t^{1 / p^{r}}\right)\right)$ is bounded for all primes $p$ and all $r \geq 1$.

\section{Acknowledgements}

Ulmer's research was partially supported by NSF grant DMS 0701053.

\section{References}

[Ber08] L. Berger, Towers of surfaces dominated by products of curves and elliptic curves of large rank over function fields, J. Number Theory 128 (2008), 3013-3030.

[Ell06] J. S. Ellenberg, Selmer groups and Mordell-Weil groups of elliptic curves over towers of function fields, Compos. Math. 142 (2006), 1215-1230.

[Ser08] J.-P. Serre, Topics in Galois theory, 2nd ed., Research Notes in Mathematics, vol. 1, A K Peters Ltd., Wellesley, MA, 2008. With notes by Henri Darmon. 
[Sch84] C.-G. Schmidt, Arithmetik abelscher Varietäten mit komplexer Multiplikation, Lecture Notes in Mathematics, vol. 1082, Springer-Verlag, Berlin, 1984 (German). With an English summary.

[Shi98] G. Shimura, Abelian varieties with complex multiplication and modular functions, Princeton Mathematical Series, vol. 46, Princeton University Press, Princeton, NJ, 1998.

[Shi86] T. Shioda, An explicit algorithm for computing the Picard number of certain algebraic surfaces, Amer. J. Math. 108 (1986), 415-432.

[Sil04] J. H. Silverman, The rank of elliptic surfaces in unramified abelian towers, J. Reine Angew. Math. 577 (2004), 153-169.

[Ulm02] D. Ulmer, Elliptic curves with large rank over function fields, Ann. of Math. (2) 155 (2002), 295-315.

[Ulm07a] L L-functions with large analytic rank and abelian varieties with large algebraic rank over function fields, Invent. Math. 167 (2007), 379-408.

[Ulm07b] Jacobi sums, Fermat Jacobians, and ranks of abelian varieties over towers of function fields, Math. Res. Lett. 14 (2007), 453-467.

[UlmDPCT] _ On Mordell-Weil groups of Jacobians over function fields, available at arXiv: 1002.3310v1.

[Xue] J. Xue, Endomorphism algebras of Jacobians of certain superelliptic curves, To appear in J. of Number Theory, available at arXiv:0902.4657v3.

[Zar03] Yu. G. Zarhin, Homomorphisms of hyperelliptic Jacobians, Tr. Mat. Inst. Steklova 241 (2003), no. Teor. Chisel, Algebra i Algebr. Geom., 90-104 (Russian, with Russian summary); English transl., Proc. Steklov Inst. Math. (2003), no. 2 (241), 79-92.

[Zar05] _ Endomorphism algebras of superelliptic Jacobians, Geometric methods in algebra and number theory, Progr. Math., vol. 235, Birkhäuser Boston, Boston, MA, 2005, pp. 339-362.

[Zar06] _ Non-isogenous superelliptic Jacobians, Math. Z. 253 (2006), 537-554.

[Zar07] _ Superelliptic Jacobians, Diophantine geometry, CRM Series, vol. 4, Ed. Norm. Pisa, 2007, pp. 363-390.

[Zar09] _ Endomorphisms of superelliptic Jacobians, Math. Z. 261 (2009), no. 3, 691707,709

School of Mathematics, Georgia Institute of Technology, Atlanta, GA, 30332, USA

E-mail address: ulmer@math.gatech.edu

Department of Mathematics, Pennsylvania State University, University Park, PA 16802, USA

E-mail address: zarhin@math.psu.edu 